\title{
Non-Hermitian scattering on a tight-binding lattice
}

\author{
Phillip C. Burke, ${ }^{1}$ Jan Wiersig, ${ }^{2}$ and Masudul Haque ${ }^{1,3}$ \\ ${ }^{1}$ Department of Theoretical Physics, Maynooth University, Maynooth, Kildare, Ireland \\ ${ }^{2}$ Institut für Physik, Otto-von-Guericke-Universität Magdeburg, Postfach 4120, D-39016 Magdeburg, Germany \\ ${ }^{3}$ Max-Planck Institute for the Physics of Complex Systems, Dresden, Germany
}

(Dated: July 20, 2020)

\begin{abstract}
We analyze the scattering dynamics and spectrum of a quantum particle on a tight-binding lattice subject to a non-Hermitian (purely imaginary) local potential. The reflection, transmission and absorption coefficients are studied as a function of the strength of this absorbing potential. The system is found to have an exceptional point at a certain strength of the potential. Unusually, all (or nearly all) of the spectrum pairs up into mutually coalescing eigenstate pairs at this exceptional point. At large potential strengths, the absorption coefficient decreases and the effect of the imaginary potential is similar to that of a real potential. We quantify this similarity by utilizing properties of a localized eigenstate.
\end{abstract}

\section{INTRODUCTION}

In recent years there has been a surge of interest in quantum systems that are described by non-Hermitian Hamiltonians. Although Hermiticity is regarded as a postulate of standard quantum mechanics, non-Hermitian Hamiltonians are useful as effective descriptions of systems where loss or gain plays an important role, such as open quantum systems [1] and optical systems described by wave equations formally analogous to a Schrödinger equation [2-4]. By now, a number of experimental platforms for the study of non-Hermitian quantum mechanics are available. These include lasers or optical resonators [5-8], coupled optical waveguides [9-13], microwave resonators [14-17] and arrays thereof [18], optical microcavities [2, 19, 20], optomechanical systems [21], photonic crystals [22, 23], acoustics [24-27] atom-cavity composites [28], exciton-polariton systems in semiconductor microcavities [29, 30], and various other arrangements [31$36]$.

Non-Hermitian Hamiltonians lead to various phenomena not present in Hermitian systems. In general, the eigenvalues of non-Hermitian Hamiltonians are complex. The left and right eigenstates of a non-Hermitian Hamiltonian are generally not equal — we confine our discussion to right eigenstates. The eigenstates are in general not mutually orthogonal. This non-orthogonality becomes extreme at points in the parameter space referred to as exceptional points [37-41]. At an exceptional point, the eigenvalues appear to become degenerate. However, it is not a genuine degeneracy as the corresponding eigenvectors coalesce as well. This results in our eigenstates no longer providing a basis spanning the entire Hilbert space. The Hamiltonian matrix is therefore non-diagonalizable and is a defective matrix [42, 43] at these exceptional points. The surviving eigenstate at an exceptional point is always chiral [44]; this chirality has been observed experimentally $[7,8,16,30,45]$. Other phenomena associated with exceptional points include loss-induced transparency [9], unidirectional transmission [26, 32, 33], lasers with non- monotonic pump-dependence [5], enhanced sensing [4648], etc. Exceptional points are also associated with the real-to-complex spectral transition for parity-time $(\mathcal{P} \mathcal{T})$ symmetric Hamiltonians [41].

In this work, we are concerned with the non-Hermitian physics of a quantum particle on a tight-binding lattice. Previous studies of non-Hermitian effects for a lattice particle include Anderson localization [49-52] and localization in quasiperiodic potentials [53, 54], invisibility (reflectionless scattering) due to non-Hermitian hopping [55] or oscillating imaginary scatterer [56], flat-band physics [57], Bloch oscillations [58], $\mathcal{P} \mathcal{T}$ symmetry obtained by combining an absorbing potential on one site with an emitting potential on another [59-65], etc. In addition, non-Hermitian tight-binding lattices form the basis of the study of non-Hermitian topological many-body systems, a topic of rapidly growing interest [66-68]. A few studies have also addressed interacting many-body systems in non-Hermitian lattice systems [69, 70].

We will consider an imaginary potential on one site of the lattice, serving as an absorbing scattering potential. This can be regarded as a lattice analog of a deltafunction scattering potential in the continuum which is purely imaginary. An imaginary scattering potential is linked to measurement [71-73], and is thus related to quantum first-passage time problems and the quantum Zeno effect [74-81]. In analogy to the quantum Zeno effect, it is expected that an imaginary potential will have suppressed absorption when the strength of the potential is large. This suggests that the absorption might be non-monotonic as a function of the strength of the dissipative potential. In this work, we explicitly show nonmonotonic dependence of the amount of absorption on the potential strength, in the context of a simple lattice model. The Hamiltonian is

$$
H=-J \sum_{j=1}^{L-1}(|j\rangle\langle j+1|+| j+1\rangle\langle j|)-i \gamma|q\rangle\langle q|,
$$

with $1 \leq q \leq L$. Here $\gamma$ is a positive constant, so that the imaginary potential is absorbing. The labels for the bra's and ket's here are site labels: The particle lives 


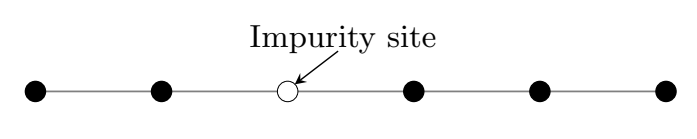

FIG. 1. The impurity is placed at one of the central sites of the lattice, as shown here for $L=6$. In this case, it could equivalently be placed on the fourth instead of the third site. For odd $L$, there is a definite central site.

on an $L$-site chain with open boundary conditions. The hopping strength will henceforth be set to $J=1$, i.e., energies and times are measured in units of $J$ and $1 / J$ respectively, and are therefore presented without units. Also, the spacing between sites is set to unity, so that lengths and wavenumbers are dimensionless as well.

The site $q$ is the location of the dissipative impurity. Since we want to study reflection and transmission, it is convenient to place the particle at the center of the lattice, at either site $\left\lfloor\frac{L}{2}\right\rfloor$ or $\left\lfloor\frac{L}{2}\right\rfloor+1$ (Fig. 1).

We present a study of the dynamics and eigenspectrum of the system (1). By scattering wavepackets numerically off the dissipative impurity, we show how the reflection, transmission and absorption fractions depend on the strength $\gamma$ of the impurity. These results are compared with the continuum problem, which is a variant of the standard textbook problem of quantum scattering off a Hermitian delta-function potential. In both cases the absorption coefficient is found to be a non-monotonic function of $\gamma$, having a maximum at a point that depends on the momentum of the incident particle or wavepacket. In addition, we present the spectrum of the Hamiltonian, which shows an unusual exceptional point at $\gamma=2$ at which all (or nearly all, depending on $L$ ) of the eigenvalues pair up. The absorption coefficient is non-monotonic and has a maximum near, but not necessary at, the exceptional point. At large $\gamma$, the absorption is vanishingly small, and the system behaves as if the impurity were a real potential $V$. In particular the system has a (anti)bound eigenstate, which allows us to draw a correspondence between values of $\gamma$ and $V$. The localized eigenstate is purely a lattice phenomenon with no analogue in the continuum.

In Section II we present the scattering results and comparisons with the continuum case. Section III discusses the spectrum and exceptional points. In Section IV we investigate the system at large $\gamma$ values, and draw a comparison between real and imaginary potentials via their bound states. In Section V we present some discussion and concluding remarks. The Appendixes present some further details on the eigenvalues and eigenstates.

\section{SCATTERING AT AN ABSORBING POTENTIAL - REFLECTION, TRANSMISSION, ABSORPTION}

In this section, we examine the scattering of a quantum particle by the dissipative impurity. To make a compar-

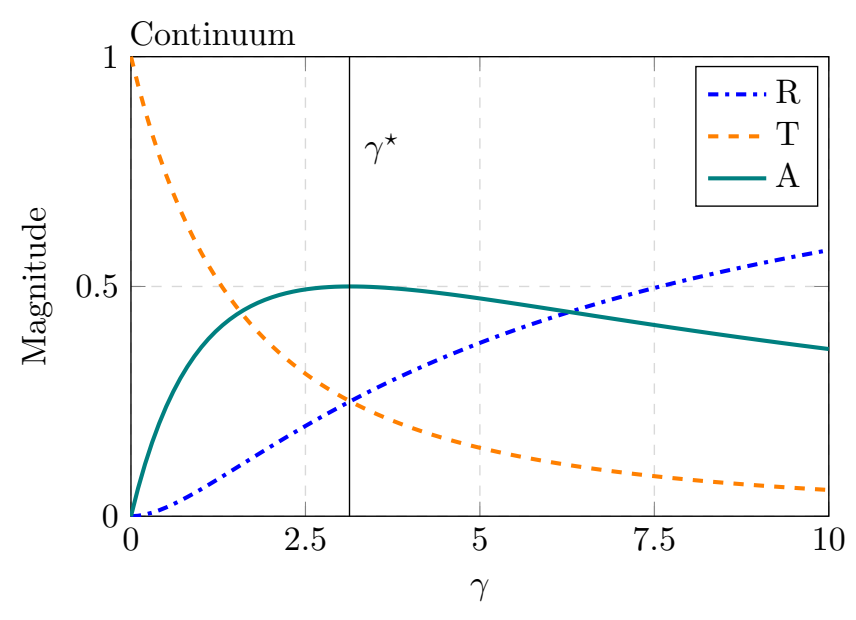

FIG. 2. Continuum scattering. The reflection, transmission and absorption probabilities $(R, T, A)$, plotted against the strength $\gamma$ of the dissipative delta-potential. Here $k=\pi / 2$, $\hbar=1$, and $m=0.5$.

ison with the corresponding continuum system, we first work out the results for the continuum system in II A, before turning back to our lattice problem in II B.

\section{A. Continuum scattering by imaginary delta-potential}

Complex scattering potentials in the continuum have been considered generally in the literature [72, 73, 82]. We are specifically interested in the case of an imaginary potential of delta-function shape, which is the analog of the single-site potential on a lattice.

In the continuum, the wavefunction $\psi(x)$ satisfies the time-independent Schrödinger Equation:

$$
-\frac{\hbar^{2}}{2 m} \frac{d^{2} \psi(x)}{d x^{2}}+V(x) \psi(x)=E \psi(x) .
$$

(We will eventually set $\hbar=1$ but retain it for now.) We take $V(x)$ to be a negative imaginary delta potential: $V(x)=-i \gamma \delta(x)$.

Solving the scattering problem is a variation of the standard textbook scattering problem with a real deltafunction potential [83]. We take the wavefunction to be of the form $e^{i k x}+r e^{-i k x}$ on the left half-line $(x<0)$ and of the form $t e^{i k x}$ on the right half-line $(x>0)$, with wavenumber $k>0$. We then use the appropriate (dis)continuity conditions at $x=0$ to solve for the reflection and transmission amplitudes $(r, t)$. This yields

$$
r=\frac{-1}{1+\frac{k \hbar^{2}}{m \gamma}} \quad, \quad t=\frac{1}{1+\frac{m \gamma}{k \hbar^{2}}} .
$$

Using (3) we can obtain the reflection, transmission, and now also absorption probability as functions of the pa- 
rameter $\gamma$ :

$$
R=|r|^{2}, \quad T=|t|^{2}, \quad A=1-R-T .
$$

We see in Fig. 2 that $R=T$ for a particular value of $\gamma$, and that $A$ is maximized by some value of $\gamma$. Using equations (3) and (4), we find that these points are both equal to

$$
\gamma^{\star}=\frac{k \hbar^{2}}{m}
$$

These expressions depend on $\hbar$ and the mass $m$. We set $\hbar=1$. To facilitate comparison with the lattice situation, we choose $m=1 / 2$ so that the quadratic dispersion $\left(\hbar^{2} k^{2} / 2 m\right)$ on the continuum matches the lowenergy part of the cosine dispersion $(-2 \cos k)$ on the lattice without impurity. Thus

$$
r=\frac{-\gamma}{\gamma+2 k}, \quad t=\frac{2 k}{\gamma+2 k}, \quad \gamma^{\star}=2 k .
$$

\section{B. Lattice}

We now turn to the lattice problem. Through numerical time evolution we will calculate the reflection and transmission fractions, $R$ and $T$, and obtain the absorption fraction using $A=1-R-T$.

We initialize our particle as a (discrete version of) a Gaussian wavepacket, localized around the site $j_{0}$ and carrying lattice momentum $k$ :

$$
|\psi(0)\rangle=\sum_{j} \psi_{j}(0)|j\rangle=\mathcal{N}^{-1} \sum_{j} e^{\frac{-\left(j-j_{0}\right)^{2}}{2 \sigma^{2}}} e^{i k j}|j\rangle
$$

where $\mathcal{N}$ is a normalization constant. A positive $k$ ensures that the wavepacket will propagate rightwards initially. The position $j_{0}$ is chosen such that the wavepacket starts on the left side of the lattice, and does not initially overlap significantly with either the lattice edges or the impurity. The width $\sigma$ is chosen to be significantly larger than 1 , but significantly smaller than $L / 2$. The wavepacket is evolved using the Hamiltonian: $|\psi(t)\rangle=e^{-i H t}|\psi(0)\rangle$. Expressing the wavefunction at time $t$ in the site basis, $|\psi(t)\rangle=\sum_{j} \psi_{j}(t)|j\rangle$, the coefficients $\psi_{j}(t)$ provide the occupancies, $\left|\psi_{j}(t)\right|^{2}$.

Fig. 3 shows the evolution of a wavepacket for three different values of $\gamma$, initially localized near the left end of a 500-site lattice. After the particle is incident on the impurity, we see different portions being reflected and transmitted. Choosing a time after the collision has occurred, such that the reflected and transmitted packets are well-separated from the impurity, one can define the coefficients based on the wavefunction coefficients at this time. The reflected (transmitted) fraction is the weight to the left (right) of the impurity. Denoting the impurity
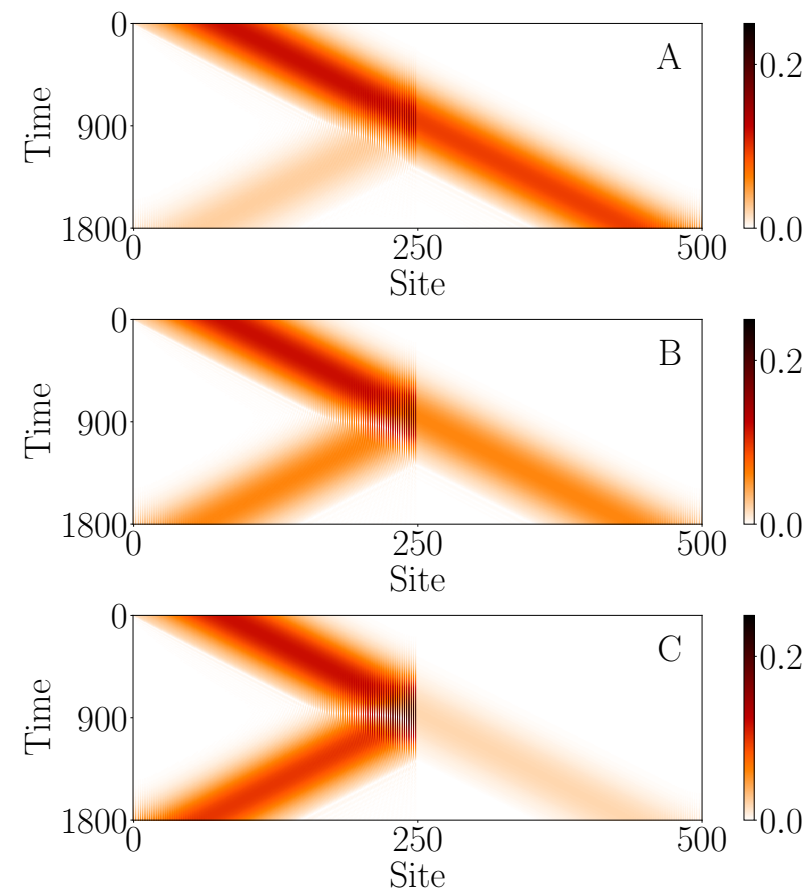

FIG. 3. Wavepacket evolution illustrated by a density plot of site occupancies $\left|\psi_{j}\right|^{2}$. Here $L=500, \sigma=40, k=\pi / 2$. A: $\gamma=0.5$ - Shows less of the wavepacket being reflected than transmitted. B: $\gamma=2$ - Shows roughly similar amounts of the wavepacket being reflected/transmitted. C: $\gamma=10$ - Shows less of the wavepacket being transmitted than reflected.

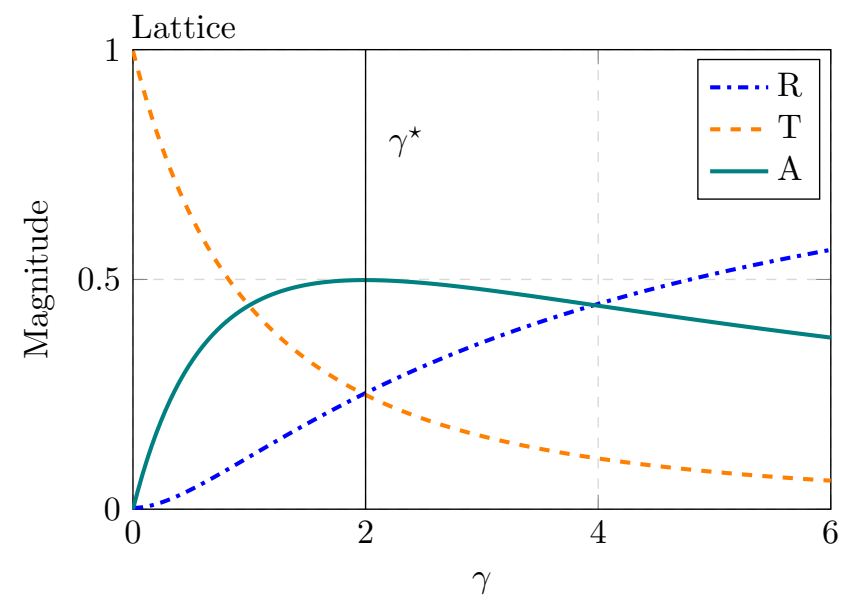

FIG. 4. Reflection, transmission and absorption probabilities calcuated using wavepacket evolution on the lattice. $(R, T, A$ plotted against $\gamma$.) Here $L=500, \sigma=40, k=\pi / 2$.

site as $q$,

$$
R=\sum_{j=1}^{q}\left|\psi_{j}\right|^{2}, \quad T=\sum_{j=q+1}^{L}\left|\psi_{j}\right|^{2}, \quad A=1-R-T .
$$

Fig. 4 shows the results of calculating the coefficients for 
a lattice with 500 sites, with the impurity at site 250, for a range of values for $\gamma$. The coefficients are extracted from time evolution with a $\sigma=40$ wavepacket. The observation of weights on the left and right parts of the lattice is performed at a time well after the wavepacket has scattered off the impurity, but well before either the reflected or the transmitted wavepacket reaches one of the boundaries. For Fig. 4, this time was $t=160$. For other values of $k$ (Fig. 5), the times are different as the speed of the wavepacket depends on $k$. We have checked that the dependence on $\sigma$ is negligible provided $1 \ll \sigma \ll L / 2$. For both Fig. 3 and Fig. 4, the wavepacket momentum is $k=\pi / 2$, for which the dispersion of the wavepacket is least severe.

\section{Comparison between Continuum and Lattice}

Comparing Figures 2 and 4, we see that our lattice results are very similar to the continuum results, except for a rescaling of $\gamma$. In the continuum case, we have found that the main feature [maximum of $A(\gamma)$, or crossing point of $R(\gamma)$ and $T(\gamma)$ ] occurs at a value of $\gamma$ that is proportional to the momentum, $\gamma^{\star}=2 k$. One therefore expects that in the lattice case $\gamma^{\star}$ should also depend on the momentum of the scattered particle. More specifically, since the single-particle dispersion changes as $k^{2} \rightarrow-2 \cos k$ in going from the continuum to lattice, one expects from the dependence of $\gamma^{\star}=2 k$ in the continuum that the dependence might be $\gamma^{\star}=2 \sin k$ on the lattice.

We can extract $\gamma^{\star}$ for various momenta by running our numerical time evolution of wavepacket scattering for various momenta and identifying the maximum of $A(\gamma)$. The results are shown in Fig. 5, comparing the continuum and lattice case. Indeed the momentum dependence of the $\gamma^{\star}$ appears to be $\approx 2 \sin k$ on the lattice, with a maximum of $\gamma^{\star} \approx 2$ for $k=\pi / 2$.

\section{SPECTRUM AND EXCEPTIONAL POINTS}

It turns out that the value $\gamma \approx 2$ also plays a special role in the spectrum of our non-Hermitian lattice Hamiltonian.

Previously we presented data for systems with 500 and 250 sites. For clarity, we now show the spectrum of smaller systems. Fig. 6 presents the eigenvalues for a system with 14 sites as a function of $\gamma$. As the eigenvalues are complex, the real and imaginary components are shown separately. We also show the 14 eigenvalues in the complex plane, for three different values of $\gamma$, in Fig. 7. For any value of $\gamma$, the real part of the eigenvalues are generally spaced between -2 and +2 , as one expects from a tight-binding one-dimensional lattice. The most visibly striking feature in the spectrum is that, at $\gamma=2$, the eigenvalues coalesce in pairs. (The coalescence is visible in the real parts - the imaginary parts are already

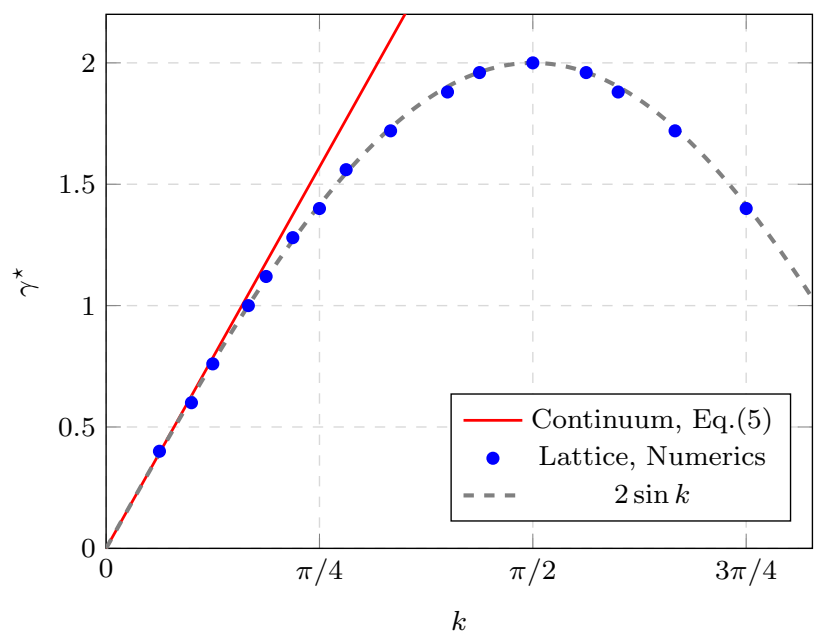

FIG. 5. Comparing results of the value of $\gamma$ for which absorption is maximised in the continuum $\left(\gamma^{\star}=2 k\right)$ and on the lattice (obtained from numerical wavepacket evolution). Lattice results are obtained with $L=250$ and $\sigma=15$. For comparison, the function $2 \sin k$ is plotted (dashed curve).

paired up even at $\gamma<2$.)

This is not a higher-order exceptional point [84-86], but rather an exceptional point where all eigenvalues pair up as second-order exceptional points, not just two eigenvalues. Of course, observing the eigenvalues is not sufficient to say that this is an exceptional point - the eigenfunctions also need to coalesce. Indeed, considering the pair of eigenstates whose eigenvalues become equal at $\gamma=2$, we find numerically that one of the eigenstates becomes equal to $-i$ times the other eigenstate.

In Appendix A we show analytically that the eigenvalues always group into degenerate pairs at $\gamma=2$, for an even- $L$ lattice with the impurity at one of the central sites. One can also show that the corresponding eigenstates for every such pair are linearly dependent.

Unlike exceptional points which separate a $\mathcal{P} \mathcal{T}$ symmetric phase from a $\mathcal{P} \mathcal{T}$-symmetry-broken phase, the eigenvalues of our system are complex on both sides of the exceptional point. The imaginary parts on average have larger magnitude near the exceptional point, and generally decrease as one moves away from $\gamma=2$, with one striking exception. The exception corresponds to one of the two eigenvalues whose real part becomes zero. The imaginary part becomes large and negative as $\gamma$ increases, and eventually becomes $\approx-\gamma$. This eigenvalue corresponds to a bound state localized at the dissipative impurity, which we will analyze in the next section.

The structure of the spectrum discussed here for $L=$ 14 is true for $L \bmod 4=2$. For other values of $L$, there are variations, which we detail in Appendix C. In particular, for odd values of $L$, there is only a single pair of eigenvalues coalescing $(L \bmod 4=3)$, or none at all $(L \bmod 4=1)$. However, even with an odd number of sites the localized eigenstate still exists for large values 

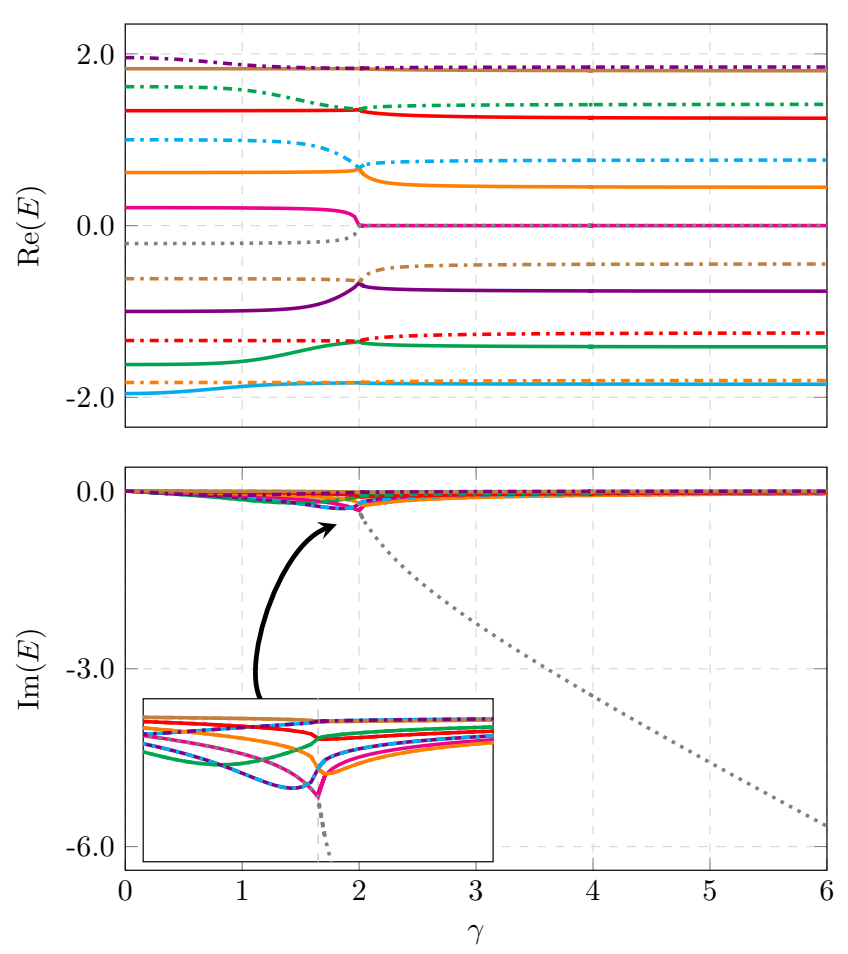

FIG. 6. Energy spectrum of the Hamiltonian (1), for $L=14$, as function of the potential strength $\gamma$. Real and imaginary parts of the eigenvalues are plotted separately.
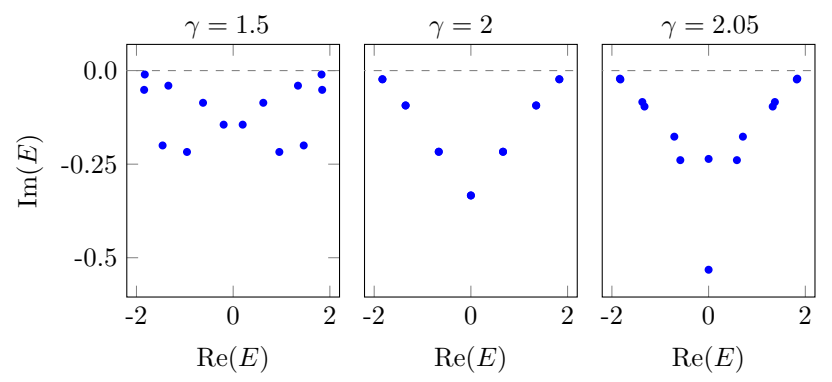

FIG. 7. Eigenvalues of the Hamiltonian (1), for $L=14$, for three values of $\gamma$, below, at and above the exceptional point. In each case, the $L$ eigenvalues are plotted on the complex plane. For $\gamma=2$, only $L / 2$ points are visible because the eigenvalues are paired.

of $\gamma$. In Appendix D we also discuss the dependence of the location of the impurity site.

\section{LARGE $\gamma$}

At large $\gamma$ the absorption decreases, suggesting that the effect of the imaginary potential is similar to that of a real potential. In this section we draw a comparison between the effects of real and imaginary on-site potentials.

In Section III we saw there was a single eigenvalue,

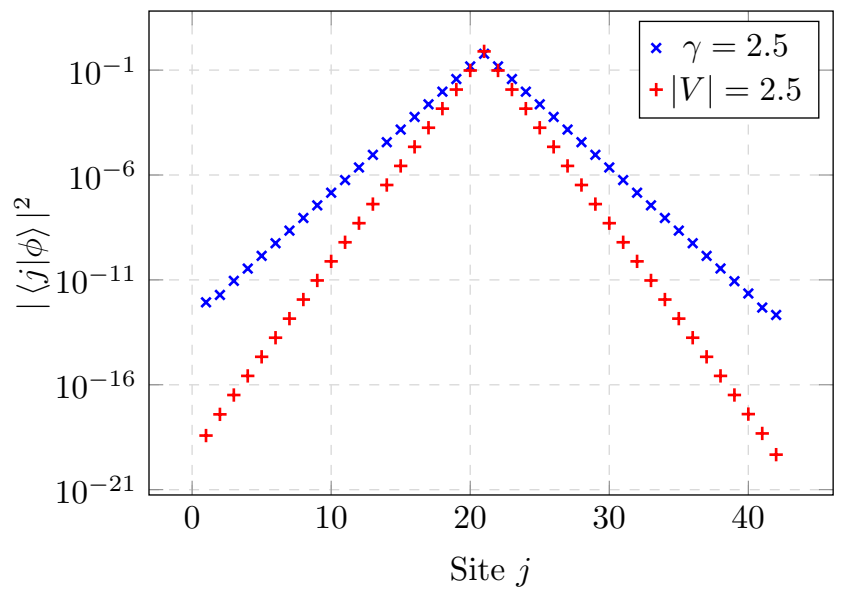

FIG. 8. Site occupancies of the localized eigenstate, for both a real $(V)$ and an imaginary $(-i \gamma)$ potential of magnitude 2.5, and $L=42$ sites. The scale is log-linear.

with a corresponding eigenstate, which had a purely imaginary negative component. At large $\gamma$ the eigenenergy approaches $-i \gamma$, for which a plausible explanation would be that the eigenstate is localized at or around the impurity site $q$ and hence its energy is primarily determined by the $-i \gamma|q\rangle\langle q|$ term in the Hamiltonian (1). Indeed the corresponding eigenstate is numerically found to be exponentially localized around the impurity site (Fig. 8).

For comparison, we also consider the effect of a real potential, i.e., the Hermitian Hamiltonian

$$
H=-J \sum_{j=1}^{L-1}(|j\rangle\langle j+1|+| j+1\rangle\langle j|)+V|q\rangle\langle q|
$$

Here $V$ is a real parameter which could be either positive or negative. It is known that this Hamiltonian supports a bound state for negative $V$ and an anti-bound state for positive $V$. (The spectrum, which is real, contains one state which separates from the band and at large $|V|$ approaches $V$.) This eigenstate is exponentially localized around site $q$.

In Fig. 8 we show the exponential localization of the eigenstate both for the real potential $(|V|=2.5)$ and for the dissipative impurity $\gamma=2.5$. At these values, the eigenstate is more strongly localized (has smaller localization length) for the case of the real potential, Eq. (9). Approximating the occupancies at site $j$ by the form $\propto e^{(j-q) / \alpha}$, where $q$ is the impurity position, one can extract the localization length $\alpha$. By extracting $\alpha$ for the localized eigenstate for various values of $\gamma$ in the case of our non-Hermitian system (1), and for various values of $V$ in the case of the system (9), we can assign to each $\gamma>2$ a value of $V$, for which the same localization length is obtained. Results of this calculation are shown in Fig. 9, for a system with $L=42$ sites. This quantifies the idea that, at large $\gamma$, an absorbing impurity behaves like a real-valued impurity. 


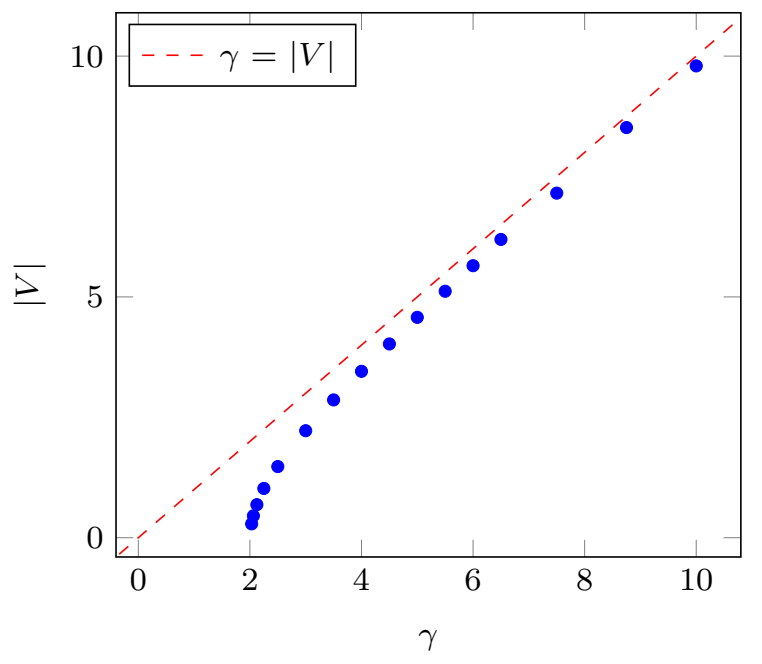

FIG. 9. A correspondence between the parameters of the real and imaginary potentials, using the localization length of the bound states, for $L=42$.

For values of $\gamma<2$, there is no bound state. For $\gamma$ slightly larger than 2 , the localization length corresponds to the bound state of a very weak real potential (very small $|V|)$. As $\gamma$ grows, the corresponding $|V|$ increases and asymptotically approaches $|V|=\gamma$. In other words, the effect of an absorbing impurity of large strength $\gamma \gg$ 2 is similar to that of a real-valued impurity of the same strength.

One can ask whether in the continuum case there is a similar correspondence - in that case also the absorption is low for the non-Hermitian model at large $\gamma$. It is wellknown that the negative real delta-potential has a single bound (localized) state. However, neither the positive real potential, nor the imaginary potential, have bound states. (If one assumes that there is a bound state for some potential, $\lambda \delta(x)$, one finds that $\lambda$ must have a real component, which is negative.) Hence no quantitative correspondence can be drawn in terms of the localization length, as we have done for the lattice.

The existence of a strongly localized eigenstate provides a simple 'spectral' interpretation of the suppression of absorption at large $\gamma$ that we have presented in Subsection II B. For large $\gamma$, the localized eigenstate has near-zero overlap with the incident wavepacket, because in the initial state the wavepacket is far from the impurity site. Thus, the wavepacket is 'shielded' from the impurity, because its dynamics is confined to the subspace of all the other eigenstates which have near-zero weight at the impurity site. Therefore the wavepacket undergoes almost no absorption. Curiously, for the suppression of absorption in the continuum case (Subsection II A), the same interpretation cannot be used, as there is no localized eigenstate in that case.

In Appendix B we show site occupancy profiles for a sample of some of the eigenstates. Other than the special (localized) eigenstate, the other eigenstates resemble those for a real potential - the eigenstates at the bottom and top of the band have few nodes, while those near the center of the band have many nodes.

\section{DISCUSSION AND CONTEXT}

We have studied the scattering dynamics and the spectrum of a tight-binding single-particle system with a nonHermitian absorbing impurity at one site, focusing on the case where the impurity is near the center of the lattice.

Setups loosely similar to ours have been explored in a few other recent works. In Ref. [56], scattering off a localized lattice impurity is studied, in the case where the strength and phase of the impurity are oscillating. Scattering was studied using Gaussian wavepackets, as in the present work. For certain parameters, the oscillatory non-Hermitian impurity was reported to allow perfect transmission ('Floquet invisibility'). In Ref. [87], the lattice impurity was placed at the lattice edge and the role of the non-orthogonality of the eigenstates on the non-unitary time evolution was explored. In addition, some related issues have been discussed in the context of $\mathcal{P} \mathcal{T}$-symmetric lattice systems formed by having imaginary potentials on multiple sites [59-65]. The spectrum of lattices with two impurities has been studied in Refs. [60, 65]. Ref. [59] reported an eigenstate which is localized on the two impurity sites - this may be considered a $\mathcal{P} \mathcal{T}$-symmetric version of the localized eigenstate we have studied. Refs. [60, 61] have made comparisons between the non-Hermitian system and corresponding Hermitian system, as we have done. After the appearance of our preprint, our single-particle nonHermitian Hamiltonian has appeared in Ref. [88] as an effective Hamiltonian.

Experimentally, lattice systems with localized losses have been studied in several contexts. In the setup of Ref. [89, 90], a Bose-Einstein condensate is realized in a one dimensional optical lattice, with engineered losses on a single site acting as a local dissipative potential. Connecting single-particle results such as ours to many-boson physics in such a setup remains an interesting challenge for future work.

A realization more similar to the single particle tightbinding system considered in this work is that with photonic lattice systems, such as those in Refs. [91, 92]. In this setup, photonic lattices are realized using femtosecond laser writing to inscribe waveguide arrays with appropriate index profiles in fused silica. The physics of photons in such an architecture can be well-described by a tight-binding model, with an additional spatial direction taking the role of time. This setup, or its variants, has been used to demonstrate a number of paradigmatic tight-binding phenomena, including Bloch oscillations [93] and Anderson localization [94, 95]. Both onedimensional and two-dimensional lattices have been realized, and lossy sites and other types of non-Hermiticity have been explored $[12,92,96]$. It is possible to cre- 
ate localized excitations (wavepackets) and observe their propagation [95, 97]. Thus, studies of scattering off lossy sites should be possible in such a setup.

Another possible experimental setting for observing scattering off non-Hermitian potentials in a tight-binding lattice might be microwave realizations using coupled dielectric resonators, such as that discussed in [18]. This setup is well approximated by a nearest-neighbour tightbinding Hamiltonian. The resonance frequency of an isolated resonator, and the coupling strength between two resonators (due to the evanescent electromagnetic field), correspond to the on-site energy and to the hopping term, respectively. A controllable on-site loss is created by placing an absorbing material on a particular resonator.

In the present work, by explicit time evolution starting from initial states which are momentum-carrying wavepackets, we found the reflection, transmission and absorption coefficients $(R, T, A)$ as a function of the impurity strength $\gamma$ and of the incident momentum $k$. The absorption was shown to first increase and then decrease as the strength $\gamma$ is increased. It can be argued that this non-monotonic behavior is related to the quantum Zeno effect. The experimental non-monotonic behavior of Ref. [34] can be interpreted in the same light. We have demonstrated and analyzed the effect in a simple lattice setting. We have also compared with the scattering of a single particle in a continuum from an absorptive deltapotential.

We have also presented the spectrum of the nonHermitian system. The system we focus on - even number of sites, impurity at one of the central sites - has an unusual exceptional point structure. At the same value of $\gamma$, all the eigenstates of the systems coalesce in pairs. This is not a higher-order of exceptional point [84-86], rather, it is a collection of many second-order coalescences at the same point in parameter space. At larger $\gamma$, the spectrum contains one localized eigenstate. This is another way in which a strong absorptive impurity acts like a real-valued impurity potential. This feature is particular to the lattice as there are no bound states in the corresponding continuum problem. The eigenvalue corresponding to the localized eigenstate has a purely imaginary value.

Our work opens up several avenues of research. We have explored scattering dynamics. A detailed study of other types of dynamics remains to be done, not only for tight-binding lattices, but also for continuum particles subjected to localized absorbers. Extending such dynamical considerations to nonlinear cases [76, 90, 98] also deserves further exploration. The spectral part of the present study provides motivation for a more thorough investigation of the spectrum of relatively simple non-Hermitian models. The structure we have found many pairs coalescing at the same point - suggests that non-Hermitian spectra may hold more surprises not yet known in the literature.

\section{ACKNOWLEDGMENTS}

We thank S. Nulty (Maynooth) for analytically demonstrating the degeneracy at $\gamma=2$ (Appendix A). PCB thanks Maynooth University (National University of Ireland, Maynooth) for funding provided via the John \& Pat Hume Scholarship.

\section{Appendix A: Analytical expressions for spectrum}

In the main text, we have shown numerically that the eigenvalues of our system coalesce in pairs at $\gamma=2$, for even $L$, when the impurity site $q$ is one of the central sites, i.e., when $q=L / 2$ or $q=(L / 2)+1$. In this Appendix, we analyze the eigenvalues analytically. We express the characteristic polynomial (whose roots are the eigenvalues) in a form which allows us to predict, first, that all the eigenvalues pair up when $q$ is one of the central sites, and second, that this is a multiple exceptional point because each eigenstate pair is linearly dependent. The characteristic polynomial is treated in Section A 1 and the case of $q=L / 2$ (or $q=L / 2+1)$ is considered in Section A 2.

\section{General location, $q$}

We want to find the eigenvalues of the $L \times L$ matrix

$$
\left[H_{q}\right]_{j k}=-\delta_{j, k+1}-\delta_{j+1, k}-i \gamma \delta_{j q} \delta_{j k}
$$

Here $1 \leq q \leq L$. The characteristic polynomial of this matrix up to a minus sign is the determinant of the tridiagonal matrix

$$
\left(\begin{array}{cccccccc}
\lambda & 1 & 0 & \ldots & \ldots & \ldots & \ldots & \ldots \\
1 & \lambda & 1 & \ldots & \ldots & \ldots & \ldots & \ldots \\
\vdots & \vdots & \ddots & \ddots & \ddots & \ldots & \ldots & \ldots \\
\vdots & \vdots & \vdots & 1 & \lambda+i \gamma & 1 & \ldots & \ldots \\
\vdots & \vdots & \vdots & \vdots & \ddots & \ddots & \ddots & \ldots \\
\vdots & \vdots & \vdots & \vdots & \vdots & 1 & \lambda & 1 \\
\vdots & \vdots & \vdots & \vdots & \vdots & \vdots & 1 & \lambda
\end{array}\right)
$$

Now the determinants of tridiagonal matrices satisfy a recurrence relation. If $P_{n}$ is the determinant of the $n \times n$ matrix with elements

$$
A_{i j}=b_{i} \delta_{i, j+1}+c_{j} \delta_{i+1, j}+a_{i} \delta_{i j}
$$

then

$$
P_{n}=a_{n} P_{n-1}-c_{n-1} b_{n-1} P_{n-2} .
$$


This recurrence relation can be verified by determinant expansion and appears in numerous sources, e.g., is mentioned in Section 8.4 of Ref. [42]. The characteristic polynomial of $H$ (A1), i.e., the determinant of the matrix (A2), therefore satisfies

$$
\begin{aligned}
P_{n} & =\lambda P_{n-1}-P_{n-2}, \quad \text { if } n \neq q \\
P_{q} & =(\lambda+i \gamma) P_{q-1}-P_{q-2}, \quad \text { if } q>1 \\
P_{0} & =1 \\
P_{1} & =\lambda+i \gamma \delta_{q 1} .
\end{aligned}
$$

A standard method of solving such linear recurrence relations is to use the $Z$ transform. Ignoring the second line in Eq. (A5), i.e., ignoring the impurity, we can get an expression for $P_{n}$ in terms of $P_{0}, P_{1}$ and $\lambda$. Defining $F(z)=Z\left\{P_{n}\right\}$ and using a shift theorem, we get

$$
z^{2} F(z)-z^{2} P_{0}-z P_{1}=\lambda\left(z F(z)-z P_{0}\right)-F(z) .
$$

After solving for $F(z)$ and decomposing into partial fractions, one can take the inverse $Z$ transform, yielding

$$
\begin{array}{r}
P_{n}=\frac{P_{0}}{\sqrt{\lambda^{2}-4}}\left[\left(x_{+}\right)^{n+1}-\left(x_{-}\right)^{n+1}\right] \\
+\frac{P_{1}-\lambda P_{0}}{\sqrt{\lambda^{2}-4}}\left[\left(x_{+}\right)^{n}-\left(x_{-}\right)^{n}\right]
\end{array}
$$

where $x_{ \pm}(\lambda)=\frac{1}{2}\left[\lambda \pm \sqrt{\lambda^{2}-4}\right]$.

Defining:

$$
K_{n}(\lambda):= \begin{cases}\frac{1}{\sqrt{\lambda^{2}-4}}\left[\left(x_{+}\right)^{n+1}-\left(x_{-}\right)^{n+1}\right] & \text { for } n \geq 0 \\ 0 & \text { for } n<0\end{cases}
$$

we can rewrite Eq. (A7) as

$$
P_{n}=P_{0} K_{n}+\left(P_{1}-\lambda P_{0}\right) K_{n-1} .
$$

Since we have derived this ignoring the impurity, Eqs. (A7), (A9) are valid either for $q=1$, in which case $P_{1}=\lambda+i \gamma$, or for values of $n$ less than $q$.

For $q=1$, we have $P_{0}=1$ and $P_{1}=\lambda+i \gamma$ so that $P_{n}=K_{n}+i \gamma K_{n-1}$, and therefore:

$$
P_{L}=K_{L}+i \gamma K_{L-1} \quad \text { for } q=1 .
$$

We now turn to $q>1$. For $n<q$, Eqs. (A7) and (A9) are valid directly with $P_{0}=1$ and $P_{1}=\lambda$, i.e., with $P_{1}-\lambda P_{0}=0$, so that

$$
P_{n}=K_{n} \quad \text { for } q>1 \text { and } n<q \text {. }
$$

We have expressions for $P_{n}$ up to $n=q-1$, but we want $P_{L}$ and $L \geq q$. To go beyond $q$, we define a new sequence of functions $Q_{n}(\lambda)$, satisfying the same recurrence relation as $P_{n}$ (A5), except with new initial conditions: $Q_{0}=P_{q-1}$ and $Q_{1}=P_{q}=(\lambda+i \gamma) P_{q-1}-P_{q-2}$. Thus we need to solve

$$
\begin{aligned}
& Q_{n}=\lambda Q_{q-1}-Q_{q-2}, \quad Q_{0}=K_{q-1}, \\
& Q_{1}=(\lambda+i \gamma) K_{q-1}-K_{q-2} .
\end{aligned}
$$

Now we have already solved the same recurrence relation for $P_{n}$, using the $Z$ transform. The solution is $Q_{n}=$ $Q_{0} K_{n}+\left(Q_{1}-\lambda Q_{0}\right) K_{n-1}$. Therefore

$$
Q_{n}=K_{q-1} K_{n}+\left(i \gamma K_{q-1}-K_{q-2}\right) K_{n-1} .
$$

Noting that $Q_{n}(\lambda)=P_{n+q-1}(\lambda)$, the determinant of the full matrix can be found as $P_{L}(\lambda)=Q_{L-q+1}(\lambda)$. Thus

$$
P_{L}(\lambda)=K_{q-1} K_{L-q+1}+\left(i \gamma K_{q-1}-K_{q-2}\right) K_{L-q} .
$$

We now introduce a slight change of notation: We refer to this polynomial as $P_{L, q}$. In other words, the characteristic polynomial of the Hamiltonian matrix of a lattice of size $L$ and having the impurity at position $q$ will be called $P_{L, q}$. Note that Eq. (A14) reduces to Eq. (A10) for $q=1$; thus

$$
P_{L, q}=K_{q-1} K_{L-q+1}+\left(i \gamma K_{q-1}-K_{q-2}\right) K_{L-q}
$$

for all positions of the impurity, $1 \leq q \leq L$.

By binomial-expanding $\left(x_{ \pm}\right)^{n+1}$, one can show that

$$
P_{L, q}\left(-\lambda^{*}\right)=(-1)^{L} P_{L, q}(\lambda)^{*} \text {. }
$$

This shows that the zeros of $P_{L, q}$ (eigenvalues of $H$ ) are symmetric by reflection through the imaginary axis in the complex plane, since if $\lambda=a+i b$ is a zero then $-\lambda^{*}=-a+i b$ is also a zero. This symmetry is obvious from the spectra shown in Fig. 7 .

\section{Impurity at center}

We now turn to the case we have focused on in this paper: when $L$ is even and $q=L / 2$ or $q=\frac{L}{2}+1$. In this case,

$$
\begin{aligned}
P_{L, \frac{L}{2}}= & K_{\frac{L}{2}-1} K_{\frac{L}{2}+1}+\left(i \gamma K_{\frac{L}{2}-1}-K_{\frac{L}{2}-2}\right) K_{\frac{L}{2}} \\
= & K_{\frac{L}{2}-1}\left(\lambda K_{\frac{L}{2}}-K_{\frac{L}{2}-1}\right)+\left(i \gamma K_{\frac{L}{2}-1}-K_{\frac{L}{2}-2}\right) K_{\frac{L}{2}} \\
= & -\left(K_{\frac{L}{2}-1}\right)^{2}+K_{\frac{L}{2}}\left(\lambda K_{\frac{L}{2}-1}-K_{\frac{L}{2}-2}\right) \\
& \quad+i \gamma K_{\frac{L}{2}-1} K_{\frac{L}{2}} \\
= & \left(K_{\frac{L}{2}}\right)^{2}-\left(K_{\frac{L}{2}-1}\right)^{2}+i \gamma K_{\frac{L}{2}-1} K_{\frac{L}{2}} .
\end{aligned}
$$

Now precisely when $\gamma=2$, this can be written as

$$
P_{L, L / 2}=\left(K_{\frac{L}{2}}+i K_{\frac{L}{2}-1}\right)^{2}
$$

This means that every root of the polynomial is a zero of order at least 2, i.e., the eigenspectrum is doubly degenerate at $\gamma=2$. We have thus analytically derived the most prominent feature of the spectrum presented in the main text.

We now argue that, for a tridiagonal system such has ours, a coalescence of eigenvalues implies a coalescence of eigenstates, i.e., that the eigenstates corresponding to the equal eigenvalues are always linearly dependent. Consider some eigenvalue $\lambda$ and corresponding eigenvector 
$X=\left(x_{1}, x_{1}, \ldots, x_{L}\right)^{T}$. Due to the form of the matrix, all the components $x_{i}$ can be written as a function of $\lambda$ and the terms on the diagonals, times the first component $x_{1}$. If we have any two eigenvectors with the same eigenvalue $\lambda$, the functions in the eigenvectors are the same functions, and hence the eigenvectors only differ in the choice of $x_{1}$, i.e., they are linearly dependent. Thus, if there is a degeneracy at some point, the eigenvectors are linearly dependent, and hence we have an exceptional point.

\section{Appendix B: Eigenstates}

We show some eigenstates of the system, through their occupancy profiles.

Since the eigenvalues are complex, there is no particularly natural way to order them. Here we order the eigenstates based on their real component, and then by their imaginary component, from smallest to largest, i.e., $1-2 i$ comes before $1+2 i$. Fig. 10 illustrates a selection of the eigenstates of a system with $L=42$ sites. They are labeled as ' $E_{i}$ ', i.e. the eigenstate presented is the state corresponding to the $i^{\text {th }}$ eigenvalue, when ordered in the described manner.

Note that the eigenvectors coefficients $\langle j \mid \phi\rangle$ are themselves complex; we only show the occupancies $|\langle j \mid \phi\rangle|^{2}$ and not the real and imaginary parts separately. (Here $|\phi\rangle$ is the eigenvector in question and $j$ is the site index.)

\section{Appendix C: Size dependence of the spectrum}

In Fig. 6 we saw coalescence of every pair of eigenvalues at $\gamma=2$. This was for a system with $L=14$ sites, and the impurity at site $q=7$. We now outline the $L$-dependence of the spectrum. The pattern is different for odd $L$. For even $L$, there is a difference between $L$ values satisfying $L=4 n+2$ and those satisfying $L=4 n$, where $n$ is a non-negative integer.

The case $L=14$, presented in the main text, belongs to the $L=4 n+2$ sequence $(6,10,14,18, \ldots)$. In Fig. 11 we show the case of $L=30$, showing exactly the same pattern: all eigenvalues pair up in a multiple exceptional point exactly at $\gamma=2$. There are an odd number of pairs, and the eigenvalues with central real values have zero eigenvalue after the coalescence, i.e., for $\gamma>2$. One of these two eigenvalues correspond to the localized eigenstate, and has imaginary part growing with $\gamma$.

For even $L$ values satisfying $L=4 n$, the situation is very similar, with one additional structure. As proved in Appendix A for even $L$, at exactly $\gamma=2$, all eigenvalues pair up; this is true for both $L=4 n+2$ and $L=4 n$. In addition, for $L=4 n$, at a value slightly above $\gamma=$ 2 , the two eigenvalues with real values nearest to zero coalesce in an additional exceptional point, as seen in Fig. 12 for $L=8$. It is at this point, $\gamma=\gamma_{1}>2$,
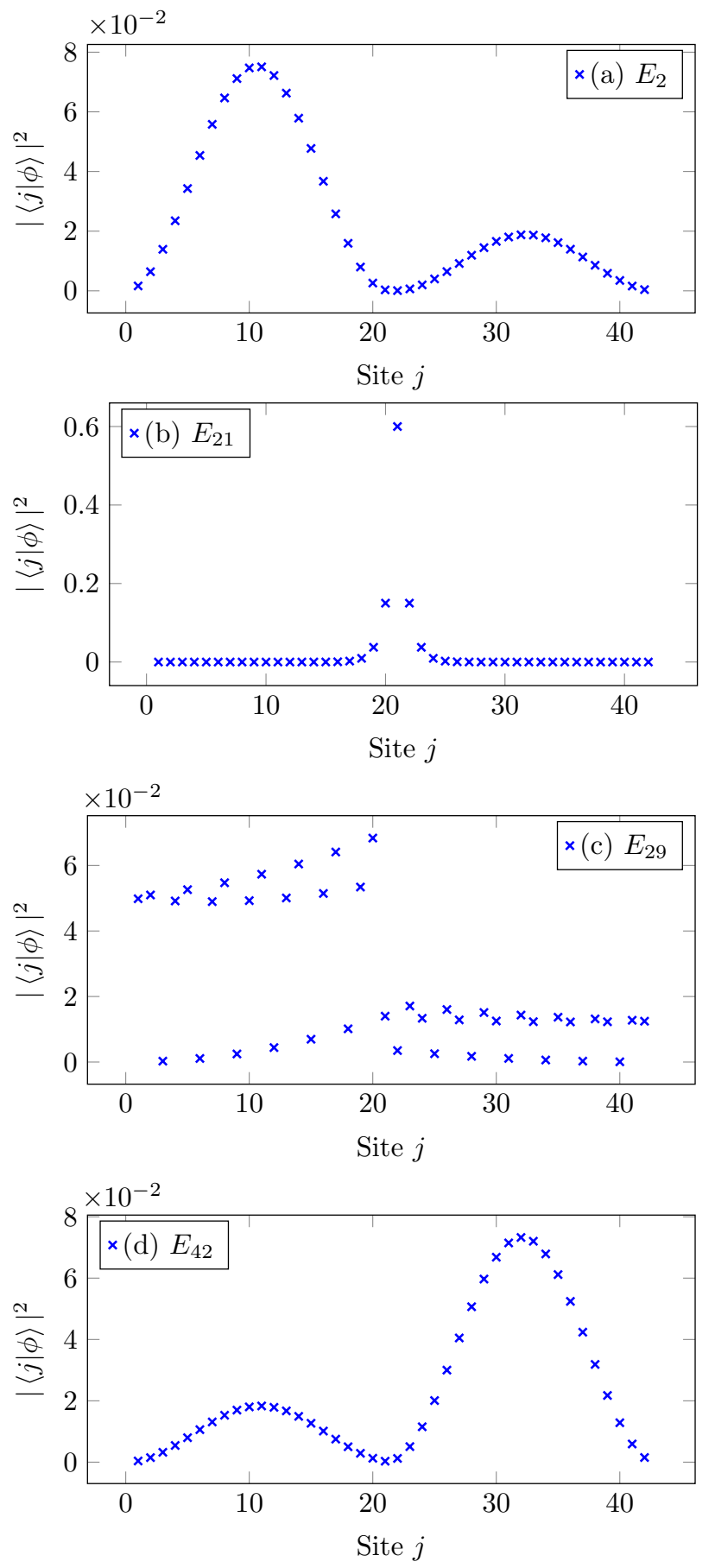

FIG. 10. Occupancy profiles of a sample of the eigenstates, for a $L=42$ system and $\gamma=2.5$. The second shown eigenstate from the top is the localized eigenstate.

that the localized state appears and the imaginary part of the corresponding eigenvalue separates off and starts to increase unboundedly in the negative direction. With increasing $L$ in the sequence $L=4 n$, the location of the new exceptional point, $\gamma_{1}$, approaches 2 .

We now turn to odd $L$, with the impurity placed on the central site, $q=(L+1) / 2$. For $L=4 n+3$, there 

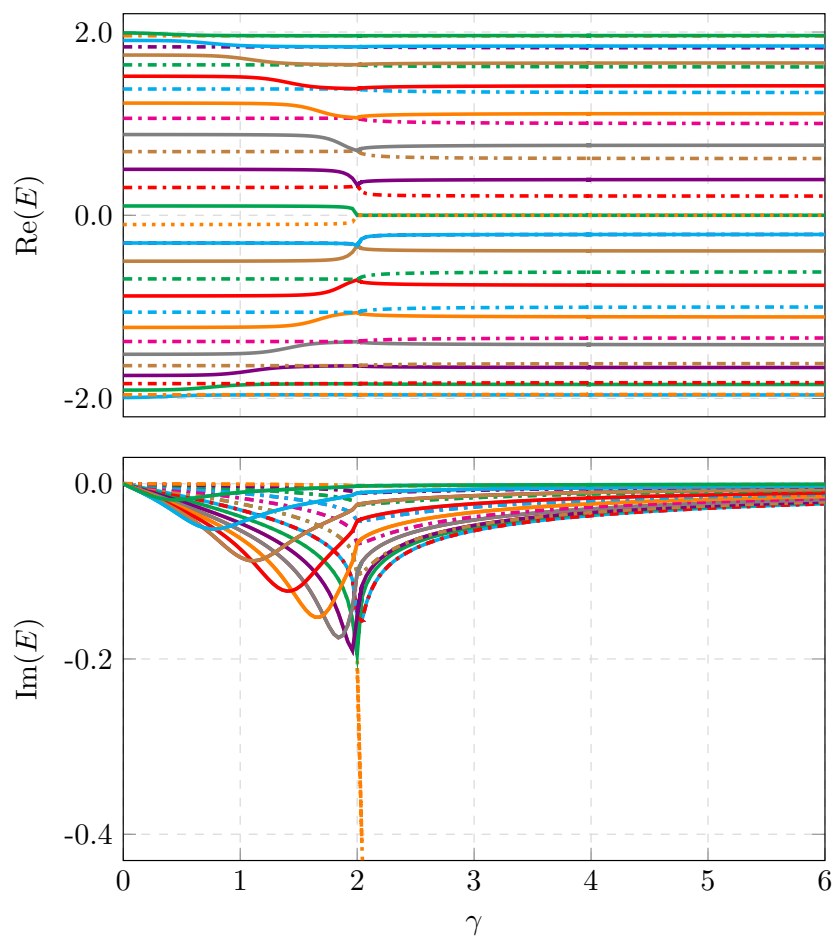

FIG. 11. Energy spectrum of a system with $L=30$. As this value is in the $L=4 n+2$ sequence, the features are the same as those described in the main text for $L=14$.
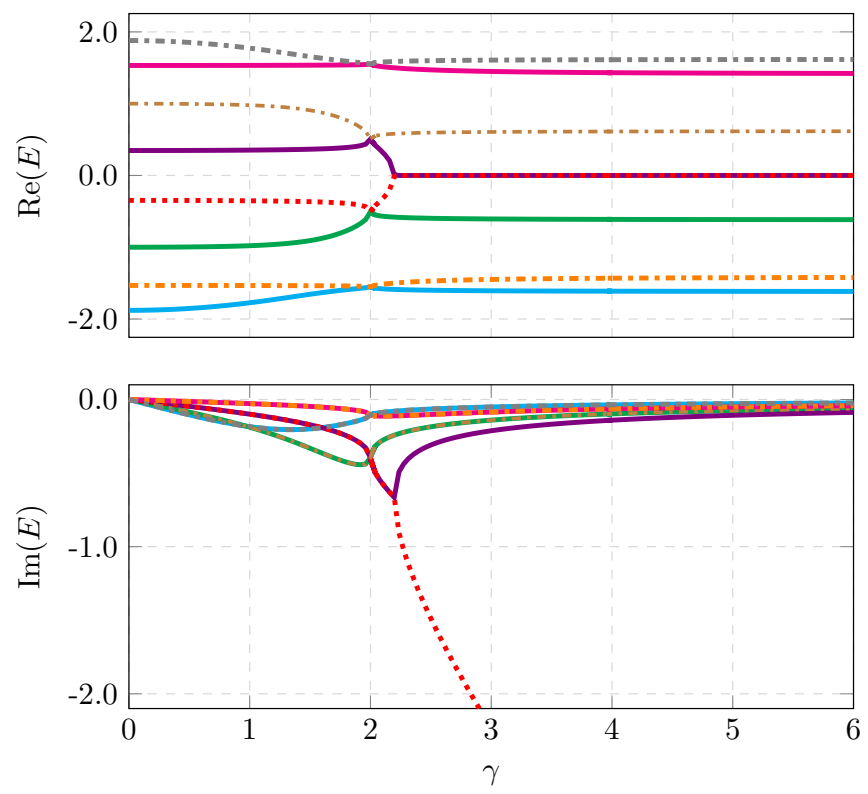

FIG. 12. Energy spectrum of a system with $L=8$. For values of $L$ in the sequence $L=4 n$, there is an extra exceptional point slightly above $\gamma=2$. The localized eigenstate appears beyond this new exceptional point.
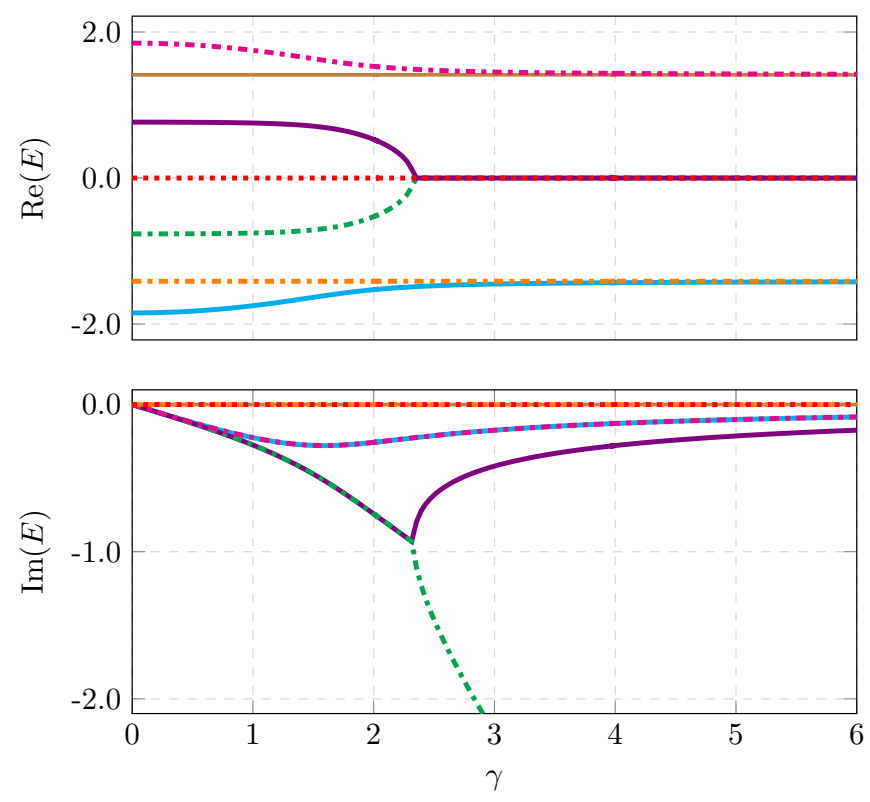

FIG. 13. Energy spectrum for $L=7$. The impurity is on the central site, $q=4$.
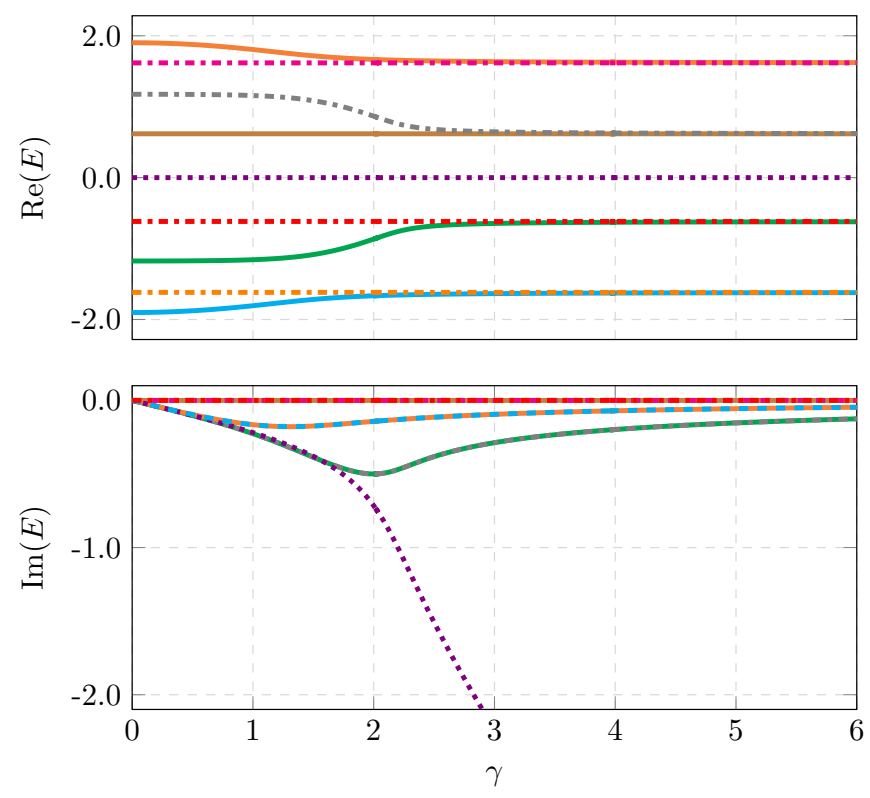

FIG. 14. Energy spectrum for $L=9$. The impurity is on the central site, $q=5$.

is only a single exceptional point. This appears to be a third-order exceptional point, and appears at a value $\gamma>2$. An example is shown in Fig. 13, for $L=7$. As the system size tends to infinity, the location of the point tends to $\gamma \rightarrow 2$. There is always a single eigenvalue that has a zero real component - the two other eigenvalues with real parts closest to zero merge with this at the exceptional point.

Finally, for $L=4 n+1$, there appears to be no exceptional points; nevertheless, at large $\gamma$ the eigenvalues 
pair up gradually. An example is shown in Fig. 14 for $L=9$. A single eigenvalue remains unpaired with zero real component. Although this does not merge with any other eigenvalue, around $\gamma \approx 2$ the imaginary component of this eigenvalue starts increasing unboundedly with $\gamma$, indicating that the corresponding eigenstate becomes localized.

In summary, although there are differences in detail between the four cases, there is always a bound state at large $\gamma$, and around $\gamma=2$ there is always some reorganization of the spectrum. With increasing $L$, the location of these features converge toward $\gamma=2$.

\section{Appendix D: Effect of impurity location}

In Appendix C, we illustrated the dependence on the lattice size $L$, focusing on the case where the impurity is located at the center of the lattice, $q=L / 2$ or $q=(L+$ 1) $/ 2$. In this Appendix we briefly discuss the dependence of the location $q$ of the impurity, focusing on the case $L=4 n+2$.

When the impurity is not on one of the central sites, the eigenvalues do not all coalesce as pairs at $\gamma=2$. As the impurity is moved from the edge site towards the center $(q=1, q=2, \ldots)$ there is an exceptional point at a value of $\gamma$ which is less than 2 for odd $q$ and larger than 2 for even $q$. At this exceptional point, the two eigenvalues with real parts closest to zero coalesce. As in the case of a centrally located impurity, when $\gamma$ is raised further beyond this value, the real parts of these two eigenvalues are locked at zero, and the imaginary part of one of this pair starts to increase in magnitude. This indicates an eigenstate localized at the impurity. (E.g., for $q=1$ this state is localized at the edge of the lattice.)
[1] I. Rotter, "A non-Hermitian Hamilton operator and the physics of open quantum systems," Journal of Physics A: Mathematical and Theoretical 42, 153001 (2009).

[2] H. Cao and J. Wiersig, "Dielectric microcavities: Model systems for wave chaos and non-Hermitian physics," Rev. Mod. Phys. 87, 61-111 (2015).

[3] S.K. Özdemir, S. Rotter, F. Nori, and L. Yang, "Paritytime symmetry and exceptional points in photonics," Nature Materials 18, 783-798 (2019).

[4] R. El-Ganainy, M. Khajavikhan, D.N. Christodoulides, and S.K. Özdemir, "The dawn of non-Hermitian optics," Communications Physics 2, 37 (2019).

[5] M. Liertzer, Li Ge, A. Cerjan, A. D. Stone, H. E. Türeci, and S. Rotter, "Pump-induced exceptional points in lasers," Phys. Rev. Lett. 108, 173901 (2012).

[6] M. Brandstetter, M. Liertzer, C. Deutsch, P. Klang, J. Schöberl, H.E. Türeci, G. Strasser, K. Unterrainer, and S. Rotter, "Reversing the pump dependence of a laser at an exceptional point," Nature Comm. 5, 4034 (2014).

[7] B. Peng, Ş.K. Özdemir, M. Liertzer, W. Chen, J. Kramer, H. Yılmaz, J. Wiersig, S. Rotter, and L. Yang, "Chiral modes and directional lasing at exceptional points," Proceedings of the National Academy of Sciences 113, 6845-6850 (2016).

[8] P. Miao, Z. Zhang, J. Sun, W. Walasik, S. Longhi, N.M. Litchinitser, and L. Feng, "Orbital angular momentum microlaser," Science 353, 464-467 (2016).

[9] A. Guo, G. J. Salamo, D. Duchesne, R. Morandotti, M. Volatier-Ravat, V. Aimez, G. A. Siviloglou, and D. N. Christodoulides, "Observation of $\mathcal{P} \mathcal{T}$-symmetry breaking in complex optical potentials," Phys. Rev. Lett. 103, 093902 (2009).

[10] C. E. Rüter, K. G. Makris, R. El-Ganainy, D. N. Christodoulides, M. Segev, and D. Kip, "Observation of paritytime symmetry in optics," Nature Physics 6 (2010), $10.1038 /$ nphys 1515 .

[11] B. Alfassi, O. Peleg, N. Moiseyev, and M. Segev, "Diverging rabi oscillations in subwavelength photonic lat- tices," Phys. Rev. Lett. 106, 073901 (2011).

[12] J.M. Zeuner, M.C. Rechtsman, Y. Plotnik, Y. Lumer, S. Nolte, M.S. Rudner, M. Segev, and A. Szameit, "Observation of a topological transition in the bulk of a nonHermitian system," Phys. Rev. Lett. 115, 040402 (2015).

[13] A. Cerjan, S. Huang, M. Wang, Kevin P. Chen, Y. Chong, and M.C. Rechtsman, "Experimental realization of a weyl exceptional ring," Nature Photonics 13, 623628 (2019).

[14] E. Persson, I. Rotter, H.-J. Stöckmann, and M. Barth, "Observation of resonance trapping in an open microwave cavity," Phys. Rev. Lett. 85, 2478-2481 (2000).

[15] C. Dembowski, H.-D. Gräf, H. L. Harney, A. Heine, W. D. Heiss, H. Rehfeld, and A. Richter, "Experimental observation of the topological structure of exceptional points," Phys. Rev. Lett. 86, 787-790 (2001).

[16] C. Dembowski, B. Dietz, H.-D. Gräf, H. L. Harney, A. Heine, W. D. Heiss, and A. Richter, "Observation of a chiral state in a microwave cavity," Phys. Rev. Lett. 90, 034101 (2003).

[17] J. Doppler, A.A. Mailybaev, J. Böhm, U. Kuhl, A. Girschik, F. Libisch, T.J. Milburn, P. Rabl, N. Moiseyev, and S. Rotter, "Dynamically encircling an exceptional point for asymmetric mode switching," Nature 537, 76 (2016).

[18] C. Poli, M. Bellec, U. Kuhl, F. Mortessagne, and H. Schomerus, "Selective enhancement of topologically induced interface states in a dielectric resonator chain," Nature Comm. 6, 6710 (2015).

[19] W. Chen, S.K. Özdemir, G. Zhao, J. Wiersig, and L. Yang, "Exceptional points enhance sensing in an optical microcavity," Nature 548, 192 (2017).

[20] Chang-Hwan Yi, Julius Kullig, and Jan Wiersig, "Pair of exceptional points in a microdisk cavity under an extremely weak deformation," Phys. Rev. Lett. 120, 093902 (2018).

[21] H. Xu, D. Mason, L. Jiang, and J.G.E. Harris, "Topological energy transfer in an optomechanical system with exceptional points," Nature 537, 80 (2016).

[22] B. Zhen, C. W. Hsu, Y. Igarashi, L. Lu, I. Kaminer, 
A. Pick, S.-L. Chua, J.D. Joannopoulos, and M. Soljacic, "Spawning rings of exceptional points out of dirac cones," Nature 525, 354-358 (2015).

[23] H. Zhou, C. Peng, Y. Yoon, C.W. Hsu, K.A. Nelson, L. Fu, J.D. Joannopoulos, M. Soljačić, and B. Zhen, "Observation of bulk fermi arc and polarization half charge from paired exceptional points," Science 359, 1009-1012 (2018).

[24] X. Zhu, H. Ramezani, C. Shi, J. Zhu, and X. Zhang, "P $\mathcal{T}$-symmetric acoustics," The Journal of the Acoustical Society of America 137, 2403-2403 (2015).

[25] R. Fleury, D. Sounas, and A. Alu, "An invisible acoustic sensor based on parity-time symmetry," Nature communications 6, 5905 (2015).

[26] C. Shi, M. Dubois, Y. Chen, L. Cheng, H. Ramezani, Y. Wang, and X. Zhang, "Accessing the exceptional points of parity-time symmetric acoustics," Nature communications 7, 11110 (2016).

[27] K. Ding, G. Ma, M. Xiao, Z.Q. Zhang, and C.T. Chan, "Emergence, coalescence, and topological properties of multiple exceptional points and their experimental realization," Phys. Rev. X 6, 021007 (2016).

[28] Y. Choi, S. Kang, S. Lim, W. Kim, J.-R. Kim, J.-H. Lee, and K. An, "Quasieigenstate coalescence in an atom-cavity quantum composite," Phys. Rev. Lett. 104, 153601 (2010).

[29] T. Gao, E. Estrecho, K.Y. Bliokh, T.C.H. Liew, M.D. Fraser, S. Brodbeck, M. Kamp, C. Schneider, S. Höfling, Y. Yamamoto, Y.S. Nori, F. Kivshar, A. Truscott, R. Dall, and E.A. Ostrovskaya, "Observation of nonHermitian degeneracies in a chaotic exciton-polariton billiard," Nature 526, 554 (2015).

[30] T. Gao, G. Li, E. Estrecho, T. C. H. Liew, D. ComberTodd, A. Nalitov, M. Steger, K. West, L. Pfeiffer, D. W. Snoke, A. V. Kavokin, A. G. Truscott, and E. A. Ostrovskaya, "Chiral modes at exceptional points in excitonpolariton quantum fluids," Phys. Rev. Lett. 120, 065301 (2018).

[31] H. Cartarius, J. Main, and G. Wunner, "Exceptional points in atomic spectra," Phys. Rev. Lett. 99, 173003 (2007).

[32] Z. Lin, H. Ramezani, T. Eichelkraut, T. Kottos, H. Cao, and D.N. Christodoulides, "Unidirectional invisibility induced by $\mathcal{P} \mathcal{T}$-symmetric periodic structures," Phys. Rev. Lett. 106, 213901 (2011).

[33] A. Regensburger, C. Bersch, M.-A. Miri, G. Onishchukov, D. N. Christodoulides, and U. Peschel, "Parity-time synthetic photonic lattices," Nature $\mathbf{4 8 8}$, 167-171 (2012).

[34] G. Barontini, R. Labouvie, F. Stubenrauch, A. Vogler, V. Guarrera, and H. Ott, "Controlling the dynamics of an open many-body quantum system with localized dissipation," Phys. Rev. Lett. 110, 035302 (2013).

[35] Y.-K. Lu, P. Peng, Q.-T. Cao, D. Xu, J. Wiersig, Q. Gong, and Y.-F. Xiao, "Spontaneous t-symmetry breaking and exceptional points in cavity quantum electrodynamics systems," Science Bulletin 63, 1096-1100 (2018).

[36] J. Li, A.K. Harter, J. Liu, L. de Melo, Y.N. Joglekar, and L. Luo, "Observation of parity-time symmetry breaking transitions in a dissipative floquet system of ultracold atoms," Nature communications 10, 855 (2019).

[37] T. Kato, Perturbation Theory for Linear Operators (Springer-Verlag Berlin Heidelberg, 1995).
[38] W.D. Heiss, "Exceptional points of non-Hermitian operators," Journal of Physics A: Mathematical and General 37, 2455-2464 (2004).

[39] M.V. Berry, "Physics of non-Hermitian degeneracies," Czechoslovak Journal of Physics 54, 1039-1047 (2004).

[40] M. Müller and I. Rotter, "Exceptional points in open quantum systems," Journal of Physics A: Mathematical and Theoretical 41, 244018 (2008).

[41] W.D. Heiss, "The physics of exceptional points," Journal of Physics A: Mathematical and Theoretical 45, 444016 (2012).

[42] G.H. Golub and C.F. Van Loan, Matrix Computations, Johns Hopkins Studies in the Mathematical Sciences (Johns Hopkins University Press, 1996).

[43] D.S. Watkins, Fundamentals of Matrix Computations, Pure and Applied Mathematics: A Wiley Series of Texts, Monographs and Tracts (Wiley, 2004).

[44] W.D. Heiss and H.L. Harney, "The chirality of exceptional points," The European Physical Journal D Atomic, Molecular, Optical and Plasma Physics 17, 149151 (2001).

[45] S. Liu, J. Wiersig, W. Sun, Y. Fan, L. Ge, J. Yang, S. Xiao, Q. Song, and H. Cao, "Transporting the optical chirality through the dynamical barriers in optical microcavities," Laser \& Photonics Reviews 12, 1800027 (2018).

[46] J. Wiersig, "Enhancing the sensitivity of frequency and energy splitting detection by using exceptional points: Application to microcavity sensors for single-particle detection," Phys. Rev. Lett. 112, 203901 (2014).

[47] J. Wiersig, "Sensors operating at exceptional points: General theory," Phys. Rev. A 93, 033809 (2016).

[48] H. Hodaei, A.U. Hassan, S. Wittek, H. Garcia-Gracia, R. El-Ganainy, D.N. Christodoulides, and M. Khajavikhan, "Enhanced sensitivity at higher-order exceptional points," Nature 548, 187 (2017).

[49] N. Hatano and D.R. Nelson, "Localization transitions in non-Hermitian quantum mechanics," Phys. Rev. Lett. 77, 570-573 (1996).

[50] I.Ya. Goldsheid and B.A. Khoruzhenko, "Distribution of eigenvalues in non-Hermitian anderson models," Phys. Rev. Lett. 80, 2897-2900 (1998).

[51] J. Heinrichs, "Eigenvalues in the non-Hermitian anderson model," Phys. Rev. B 63, 165108 (2001).

[52] Giovanni Barontini and Vera Guarrera, "Localization by dissipative disorder: Deterministic approach to position measurements," Phys. Rev. A 91, 032114 (2015).

[53] S. Longhi, "Topological phase transition in nonHermitian quasicrystals," Phys. Rev. Lett. 122, 237601 (2019).

[54] S. Longhi, "Metal-insulator phase transition in a nonHermitian aubry-andré-harper model," Phys. Rev. B 100, 125157 (2019).

[55] S. Longhi, "Invisibility in non-Hermitian tight-binding lattices," Phys. Rev. A 82, 032111 (2010).

[56] S. Longhi, "Non-Hermitian Floquet invisibility," EPL (Europhysics Letters) 117, 10005 (2017).

[57] D. Leykam, S. Flach, and Y.D. Chong, "Flat bands in lattices with non-Hermitian coupling," Phys. Rev. B 96, 064305 (2017).

[58] S. Longhi, "Bloch oscillations in complex crystals with $\mathcal{P} \mathcal{T}$ symmetry," Phys. Rev. Lett. 103, 123601 (2009).

[59] O. Bendix, R. Fleischmann, T. Kottos, and B. Shapiro, "Exponentially fragile $\mathcal{P} \mathcal{T}$ symmetry in lattices with 
localized eigenmodes," Phys. Rev. Lett. 103, 030402 (2009).

[60] L. Jin and Z. Song, "Solutions of $\mathcal{P} \mathcal{T}$-symmetric tightbinding chain and its equivalent Hermitian counterpart," Phys. Rev. A 80, 052107 (2009).

[61] L. Jin and Z. Song, "Physics counterpart of the $\mathcal{P} \mathcal{T}$ nonHermitian tight-binding chain," Phys. Rev. A 81, 032109 (2010).

[62] Y. N. Joglekar, D. Scott, M. Babbey, and A. Saxena, "Robust and fragile $\mathcal{P} \mathcal{T}$-symmetric phases in a tightbinding chain," Phys. Rev. A 82, 030103 (2010).

[63] L. Jin and Z. Song, "Parity-time symmetry under magnetic flux," Phys. Rev. A 93, 062110 (2016).

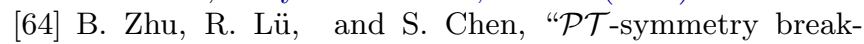
ing for the scattering problem in a one-dimensional non-Hermitian lattice model," Phys. Rev. A 93, 032129 (2016).

[65] A. Ortega, T. Stegmann, Benet. L., and H. Larralde, " $\mathcal{P} \mathcal{T}$-symmetric tight-binding chain with gain and loss: A completely solvable model," (2019), arXiv:1906.10116v1 [quant-ph].

[66] T. Ozawa, H. M. Price, A. Amo, N. Goldman, M. Hafezi, L. Lu, M.C. Rechtsman, D. Schuster, J. Simon, O. Zilberberg, and I. Carusotto, "Topological photonics," Rev. Mod. Phys. 91, 015006 (2019).

[67] V. M. Martinez Alvarez, J. E. Barrios Vargas, M. Berdakin, and L. E. F. Foa Torres, "Topological states of non-Hermitian systems," The European Physical Journal Special Topics 227, 1295-1308 (2018).

[68] Luis E F Foa Torres, "Perspective on topological states of non-hermitian lattices," Journal of Physics: Materials 3, 014002 (2019).

[69] P. A. McClarty and J. G. Rau, "Non-Hermitian topology of spontaneous magnon decay," Phys. Rev. B 100, 100405 (2019).

[70] David J. Luitz and Francesco Piazza, "Exceptional points and the topology of quantum many-body spectra," Phys. Rev. Research 1, 033051 (2019).

[71] G.R. Allcock, "The time of arrival in quantum mechanics I. formal considerations," Annals of Physics 53, 253 - 285 (1969).

[72] G.R. Allcock, "The time of arrival in quantum mechanics II. the individual measurement," Annals of Physics 53, $286-310$ (1969).

[73] G.R. Allcock, "The time of arrival in quantum mechanics III: The measurement ensemble," Annals of Physics 53, $311-348$ (1969).

[74] B. Misra and E.C.G. Sudarshan, "The zenos paradox in quantum theory," Journal of Mathematical Physics 18, 756-763 (1977).

[75] P. Facchi and S. Pascazio, "Quantum zeno dynamics: mathematical and physical aspects," Journal of Physics A: Mathematical and Theoretical 41, 493001 (2008).

[76] D. A. Zezyulin, V. V. Konotop, G. Barontini, and H. Ott, "Macroscopic zeno effect and stationary flows in nonlinear waveguides with localized dissipation," Phys. Rev. Lett. 109, 020405 (2012).

[77] P. L. Krapivsky, J. M. Luck, and K. Mallick, "Survival of classical and quantum particles in the presence of traps," Journal of Statistical Physics 154, 1430-1460 (2014).

[78] S. Dhar, S. Dasgupta, A. Dhar, and D. Sen, "Detection of a quantum particle on a lattice under repeated projective measurements," Phys. Rev. A 91, 062115 (2015).

[79] S. Dhar, S. Dasgupta, and A. Dhar, "Quantum time of arrival distribution in a simple lattice model," Journal of Physics A: Mathematical and Theoretical 48, 115304 (2015).

[80] W. Kozlowski, S. F. Caballero-Benitez, and I. B. Mekhov, "Non-Hermitian dynamics in the quantum zeno limit," Phys. Rev. A 94, 012123 (2016).

[81] H. Fröml, A. Chiocchetta, C. Kollath, and S. Diehl, "Fluctuation-induced quantum zeno effect," Phys. Rev. Lett. 122, 040402 (2019).

[82] J.G. Muga, J.P. Palao, B. Navarro, and I.L. Egusquiza, "Complex absorbing potentials," Physics Reports 395, $357-426$ (2004).

[83] D.J. Griffiths, Introduction to Quantum Mechanics (Cambridge University Press, 2017).

[84] E.-M. Graefe, U. Günther, H.J. Korsch, and A.E. Niederle, "A non-Hermitian symmetric bose-hubbard model: eigenvalue rings from unfolding higher-order exceptional points," Journal of Physics A: Mathematical and Theoretical 41, 255206 (2008).

[85] W.D. Heiss, "Chirality of wavefunctions for three coalescing levels," Journal of Physics A: Mathematical and Theoretical 41, 244010 (2008).

[86] G. Demange and E.-M. Graefe, "Signatures of three coalescing eigenfunctions," Journal of Physics A: Mathematical and Theoretical 45, 025303 (2011).

[87] J. Wiersig, "Role of nonorthogonality of energy eigenstates in quantum systems with localized losses," Phys. Rev. A 98, 052105 (2018).

[88] Heinrich Fröml, Christopher Muckel, Corinna Kollath, Alessio Chiocchetta, and Sebastian Diehl, "Ultracold quantum wires with localized losses: Many-body quantum Zeno effect," Phys. Rev. B 101, 144301 (2020).

[89] R. Labouvie, B. Santra, S. Heun, S. Wimberger, and H. Ott, "Negative differential conductivity in an interacting quantum gas," Phys. Rev. Lett. 115, 050601 (2015).

[90] R. Labouvie, B. Santra, S. Heun, and H. Ott, "Bistability in a driven-dissipative superfluid," Phys. Rev. Lett. 116, 235302 (2016).

[91] M. Heinrich, M. Miri, S. Stüzer, R. El-Ganainy, S. Nolte, A. Szameit, and D. N. Christodoulides, "Supersymmetric mode converters," Nature Communications 5, 3698 (2014).

[92] S. Eichelkraut, T.and Weimann, M. Kremer, M. Ornigotti, and A. Szameit, "Passive $\mathcal{P} \mathcal{T}$-symmetry in laserwritten optical waveguide structures," in Parity-time Symmetry and Its Applications, edited by Demetrios Christodoulides and Jianke Yang (Springer Singapore, Singapore, 2018) pp. 123-153.

[93] R. Morandotti, U. Peschel, J. S. Aitchison, H. S. Eisenberg, and Y. Silberberg, "Experimental observation of linear and nonlinear optical Bloch oscillations," Phys. Rev. Lett. 83, 4756-4759 (1999).

[94] T. Schwartz, G. Bartal, S. Fishman, and M. Segev, "Transport and anderson localization in disordered two-dimensional photonic lattices," Nature 446, 52-55 (2007).

[95] D. Guzman-Silva, M. Heinrich, T. Biesenthal, Y.V. Kartashov, and A. Szameit, "Experimental study of the interplay between dynamic localization and Anderson localization," Opt. Lett. 45, 415-418 (2020).

[96] A. Szameit, M. C. Rechtsman, O. Bahat-Treidel, and M. Segev, " $\mathcal{P} \mathcal{T}$-symmetry in honeycomb photonic lattices," Phys. Rev. A 84, 021806 (2011).

[97] M. Heinrich, M.-A. Miri, S. Stützer, S. Nolte, D.N. 
Christodoulides, and A. Szameit, "Observation of supersymmetric scattering in photonic lattices," Opt. Lett. 39, 6130-6133 (2014).

[98] V. A. Brazhnyi, V. V. Konotop, V. M. Pérez-García, and H. Ott, "Dissipation-induced coherent structures in bose-einstein condensates," Phys. Rev. Lett. 102, 144101 (2009). 